\title{
Reduction of Pentylenetetrazole-Induced Seizure Activity in Awake Rats by Seizure-Triggered Trigeminal Nerve Stimulation
}

\author{
Erika E. Fanselow, ${ }^{1}$ Ashlan P. Reid, ${ }^{2}$ and Miguel A. L. Nicolelis ${ }^{1,2}$ \\ Departments of ${ }^{1}$ Neurobiology and ${ }^{2}$ Biomedical Engineering, Duke University Medical Center, \\ Durham, North Carolina 27710
}

\begin{abstract}
Stimulation of the vagus nerve has become an effective method for desynchronizing the highly coherent neural activity typically associated with epileptic seizures. This technique has been used in several animal models of seizures as well as in humans suffering from epilepsy. However, application of this technique has been limited to unilateral stimulation of the vagus nerve, typically delivered according to a fixed duty cycle, independently of whether ongoing seizure activity is present. Here, we report that stimulation of another cranial nerve, the trigeminal nerve, can also cause cortical and thalamic desynchronization, resulting in a reduction of seizure activity in awake rats. Furthermore, we demonstrate that providing this stimulation only when seizure activity begins results in more effective and safer seizure reduction per second of stimulation than with previous methods. Seizure activity induced by intraperitoneal injection of pentylenetetrazole was recorded from microwire electrodes in the thalamus and cortex of awake rats while the infraorbital branch of the trigeminal nerve was stimulated via a chronically implanted nerve cuff electrode. Continuous unilateral stimulation of the trigeminal
\end{abstract}

nerve reduced electrographic seizure activity by up to $78 \%$, and bilateral trigeminal stimulation was even more effective. Using a device that automatically detects seizure activity in real time on the basis of multichannel field potential signals, we demonstrated that seizure-triggered stimulation was more effective than the stimulation protocol involving a fixed duty cycle, in terms of the percent seizure reduction per second of stimulation. In contrast to vagus nerve stimulation studies, no substantial cardiovascular side effects were observed by unilateral or bilateral stimulation of the trigeminal nerve. These findings suggest that trigeminal nerve stimulation is safe in awake rats and should be evaluated as a therapy for human seizures. Furthermore, the results demonstrate that seizure-triggered trigeminal nerve stimulation is technically feasible and could be further developed, in conjunction with real-time seizure-predicting paradigms, to prevent seizures and reduce exposure to nerve stimulation.

Key words: epilepsy; trigeminal nerve; seizure detection; seizure control; pentylenetetrazole; bilateral stimulation
Seminal neurophysiological studies performed several decades ago demonstrated that stimulation of either cranial nerves or areas of the brainstem can cause desynchronization of the cortical EEG (Moruzzi and Magoun, 1949; Zanchetti et al., 1952; Magnes et al., 1961; Chase et al., 1967). Such desynchronization typically reflects a state of arousal and full vigilance in mammals and is opposite to the high degree of EEG synchronization observed during seizure activity. Building on these classical findings, several researchers showed that stimulation of the vagus nerve can lead to EEG desynchronization (Zanchetti et al., 1952; Chase et al., 1966, 1967; Chase and Nakamura, 1968). More recently, several studies have demonstrated that the desynchronization induced by vagus nerve stimulation (VNS) in dogs can be used to reduce strychnine- or pentylenetetrazole (PTZ)-induced seizure activity (Zabara, 1985, 1992). This paradigm was demonstrated subsequently to be effective in other animals, with other seizure models (Lockard et al., 1990; Woodbury and Woodbury, 1990; McLachlan, 1993), and has been used with moderate success in treating humans who have otherwise intractable epileptic seizures (Penry and Dean, 1990; Uthman et al., 1990, 1993; Ben-Menachem et al., 1994; Vagus Nerve Stimulation Study Group, 1995; McLachlan, 1997; Schachter and Saper, 1998). Because $0.5-2 \%$ of the population has epilepsy (Schachter and Saper, 1998; McNamara, 1999) and 10-50\% of these patients do not respond well to antiepileptic medications and/or may not be candidates for resective epilepsy surgery (McLachlan, 1997; Schachter and Saper, 1998), there is a substan-

\footnotetext{
Received May 19, 2000; revised Aug. 7, 2000; accepted Aug. 11, 2000.

This work was funded by a grant from the Klingenstein Foundation to M.A.L.N. and by National Institute of Dental Research Grant DE-11121-01 to M.A.L.N. We thank Dr. James O. McNamara for helpful comments on this manuscript.

Correspondence should be addressed to Erika E. Fanselow, Department of Neurobiology, Duke University Medical Center, Durham, NC 27710. E-mail: efanse@neuro.duke.edu.

Copyright (C) 2000 Society for Neuroscience $0270-6474 / 00 / 208160-09 \$ 15.00 / 0$
}

tial need for potential alternative therapies for chronic seizures. Indeed, the VNS technique has recently received FDA approval and is currently being used in patients.

There are, however, several limiting factors of the VNS technique, which, if addressed, could greatly increase the efficacy and applicability of cranial nerve stimulation for seizure reduction in patients. First, the standard implementation of VNS in humans typically involves stimulating the vagus nerve on a fixed, intermittent duty cycle (e.g., $30 \mathrm{sec}$ on; 5 min off; $24 \mathrm{hr}$ a day), independently of whether any seizure activity is ongoing or imminent (although the use of manual patient- or caregiver-triggered stimulation via a handheld magnet has also been used) (Terry et al., 1990; Uthman et al., 1993). This type of protocol has been used in previous studies for two main reasons. First, although continuous stimulation may have a greater therapeutic effect than intermittent stimulation (Takaya et al., 1996), continuous stimulation can cause nerve damage, whereas intermittent stimulation does not (Agnew et al., 1989; Agnew and McCreery, 1990; Ramsay et al., 1994). Second, the side effects associated with VNS are typically experienced during the stimulation (Uthman et al., 1993; Ramsay et al., 1994; McLachlan, 1997), so giving intermittent stimulation reduces their occurrence. However, because stimulation is delivered regardless of whether seizure activity is present or is likely to occur, this fixed stimulation protocol has the disadvantage that the patient may receive excess stimulation.

The second main problem is that the vagus nerve is involved in, among other things, cardiovascular and abdominal visceral functions. Indeed, because of the pattern of vagus innervation of the heart, the vagus nerve can only safely be stimulated unilaterally (i.e., on the left side only). This is a potential limitation in the efficacy of cranial nerve stimulation because the effects of the stimulation may be bilateral (Chase et al., 1966; Zabara, 1992; Henry et al., 1998, 1999) and may, therefore, be aided by adding more stimulation sites. For these reasons, use of a nerve without 


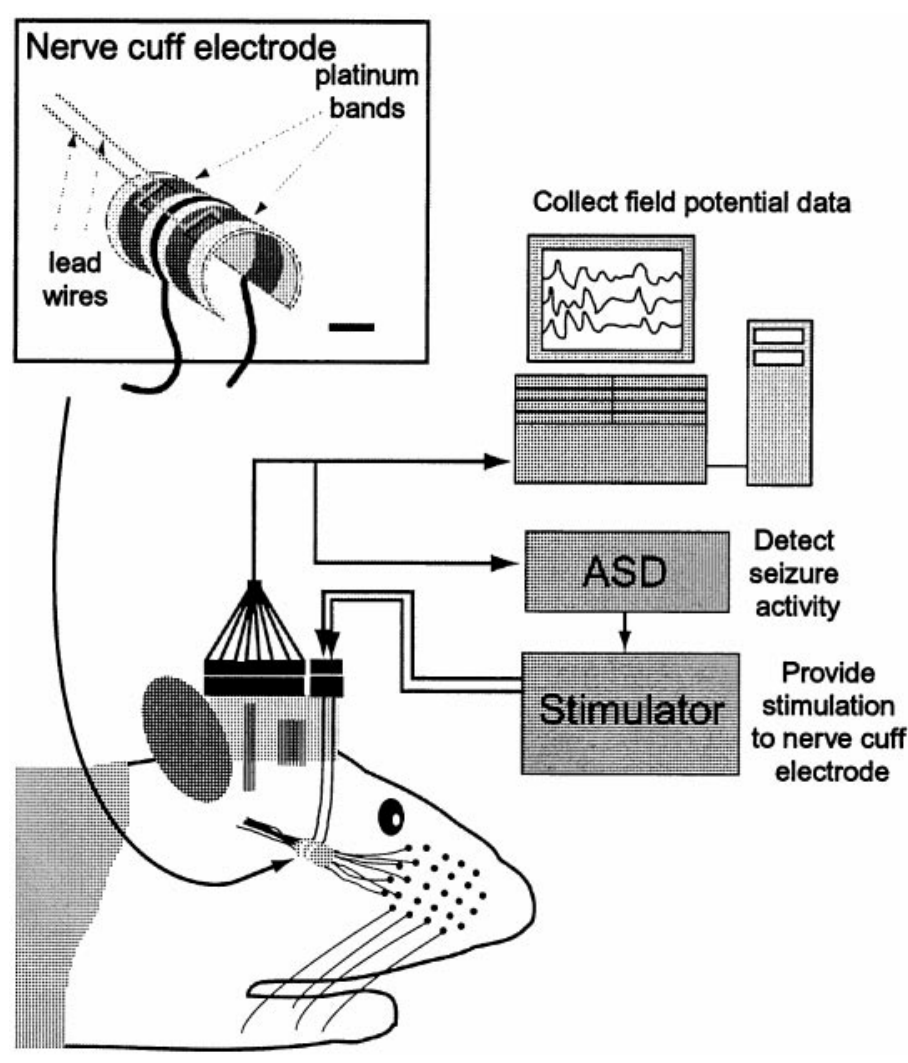

Figure 1. Schematic drawing of nerve cuff electrode and ASD device. Field potential signals from chronically implanted microwires in the VPM thalamus and/or SI cortex were sent to an amplifier and recording unit for collection, as well as to the ASD device. When the ASD device detected seizure activity, it sent a signal to the stimulator, which delivered a current pulse to the implanted nerve cuff electrode. Scale bar: inset, $1 \mathrm{~mm}$.

the types of visceral fibers that are found in the vagus nerve could potentially be more effective for seizure reduction.

Here, we demonstrate that trigeminal nerve stimulation reduced PTZ-induced seizures in awake rats. In addition, we show that such stimulation was more effective when it was bilateral. Finally, we describe a real-time interface for automatically detecting seizure activity and eliminating it by providing stimulation only when seizure activity is present. These results suggest that if such techniques were implemented in human patients, they could greatly decrease the amount of stimulation necessary for seizure control while increasing the efficacy of cranial nerve stimulation as a therapy for intractable epilepsy.

\section{MATERIALS AND METHODS}

Subjects. Eight adult female Long-Evans hooded rats weighing between 230 and $375 \mathrm{gm}$ served as subjects in this study. All procedures and experiments were conducted in compliance with Duke University Medical Center animal use policies and were approved by the Duke University Institutional Animal Care and Use Committee.

Induction of seizures. Seizures were induced by intraperitoneal injection of PTZ $(40 \mathrm{mg} / \mathrm{kg})$. This dose of PTZ induced generalized seizure activity for 1-2 hr. This seizure activity was manifested in two ways: (1) highly synchronous, large-amplitude activity in the thalamic and cortical field potential traces (see Figs. 2, 3, 5-7) and (2) clonic jerking of the body and forelimbs. These two indicators of seizure activity were highly correlated at all times, as assessed by concurrent visual inspection of the animal and the real-time field potential traces. Occasionally, a supplemental dose of PTZ $(7-10 \mathrm{mg} / \mathrm{kg})$ was given if seizures ceased in $<1 \mathrm{hr}$.

Nerve cuff electrodes. The infraorbital nerve(s) was stimulated unilaterally or bilaterally via chronically implanted nerve cuff electrodes. These electrodes were constructed in-house and consisted of two bands of platinum $(0.5 \mathrm{~mm}$ wide and $0.025 \mathrm{~mm}$ thick; $\sim 0.8 \mathrm{~mm}$ separation between bands) that ran circumferentially around the infraorbital (IO) nerve (see Fig. 1, inset). The platinum bands were held in place and electrically insulated by a thin Sylgard coating. Each band was connected to a piece of flexible, three-stranded Teflon-coated wire that was used to pass current between the bands (Fanselow and Nicolelis, 1999).
Chronic implantation of microwire electrodes. Microwire electrodes (NBLabs, Denison, TX) were chronically implanted into the ventral posterior medial thalamus (VPM) and/or primary somatosensory cortices (SI) for use in recording field potentials in these areas (Fig. 1). Three rats had arrays of 16 microwires implanted in layer V of the SI cortex and bundles of 16 electrodes implanted into the VPM thalamus, both contralateral to the stimulated nerve. Five rats had two arrays of 16 electrodes implanted, one each into layer V of the left and right SI cortices so that recordings could be made both ipsilateral and contralateral to the nerve being stimulated.

These implants were performed under pentobarbital anesthesia (50 $\mathrm{mg} / \mathrm{kg}$ ). Small craniotomies were performed over the areas into which electrodes were to be implanted [coordinates from Paxinos and Watson (1986)]. Electrodes were slowly lowered into these areas, and recordings were made throughout the implantation process to assess electrode location. After electrodes were in the correct position, they were cemented to skull screws by the use of dental acrylic (Nicolelis et al., 1997).

Chronic implantation of nerve cuff electrodes. After implantation of the microwires, nerve cuff electrodes were implanted either unilaterally or bilaterally. A dorsoventral incision was made on the face several millimeters caudal to the caudal edge of the whiskerpad. Tissue was dissected until the infraorbital nerve was exposed, and the cuff electrode was positioned around the nerve such that the nerve lay inside the cuff. The cuff was then tied around the nerve to hold it in place, and the wound was sutured. The Teflon-coated leads from the platinum bands were run subcutaneously to the top of the head where they were attached to connector pins and affixed to the skull.

Recording procedures. Field potential recordings from VPM thalamus and SI cortex were made using chronically implanted microwires (Nicolelis et al., 1997). Field potentials were collected using a Grass Model 15 amplifier and stored on a personal computer. Signals were collected at a sampling rate of $512 \mathrm{~Hz}$ and bandpass filtered during collection at 1-100 $\mathrm{Hz}$. During each recording session, 16 field potential channels were recorded, 8 from each area from which recordings were made in a given rat (either VPM and SI, or SI left and SI right). In addition, one channel was recorded for each nerve cuff being stimulated (unilateral or bilateral stimulation) to indicate when stimulation occurred. During experiments, animals were awake and allowed to move freely in a $30 \mathrm{~cm} \times 30 \mathrm{~cm}$ recording chamber.

Stimulation parameters. Stimulation of the IO nerve cuff electrodes was provided by the use of a Grass S 8800 stimulator in conjunction with a Grass SIU6 stimulus isolation unit. Unimodal square current pulses with a duration of $500 \mu \mathrm{sec}$ were given at a range of currents and frequencies. Current values were varied from 3 to $11 \mathrm{~mA}$ ( $2 \mathrm{~mA}$ intervals), and frequency values were varied from 1 to $333 \mathrm{~Hz}(1,5,10,20,50,100,125$, 200 , and $333 \mathrm{~Hz}$ ). Animals tolerated stimulation at these levels without indication of pain, although in some animals there appeared to be a sensation of pressure on the face at the highest current and frequency settings. This was evidenced by a tendency for the animals to back up when the stimulus began, in the direction away from the stimulated side if unilateral stimulation was provided or straight back in the case of bilateral stimulation. In addition, at lower stimulus intensities animals would occasionally scratch at the whiskerpad on the side of the face being stimulated during the first few seconds of stimulation. However, the scratching was neither intense nor prolonged.

Automatic seizure detection device. A device was designed and built in-house to automatically detect seizure activity in real time and immediately trigger a stimulator when a seizure was detected (Fig. 1). The automatic seizure detection (ASD) device first low-pass filtered the raw field potentials obtained from the microwire arrays at $30 \mathrm{~Hz}$. Circuitry then determined whether the field potential activity surpassed a threshold voltage value, indicative that seizure activity was present. When the field potential voltage crossed the threshold, a TTL pulse was sent to the Grass S8800 stimulator, which delivered a $0.5 \mathrm{sec}$ train of $500 \mu \mathrm{sec}$ pulses at 333 $\mathrm{Hz}$. The current level was dictated by the stimulation protocol for a given trial. Trains of stimuli were presented as long as the field potential activity remained above the threshold value (i.e., as long as seizure activity was ongoing). The train duration for the seizure-triggered stimulation $(0.5 \mathrm{sec})$ was chosen because it was the shortest duration that we found to be effective for stopping the seizure activity, and we wanted to keep the stimulation as short as possible to reduce the total amount of stimulation given. The voltage threshold was set manually for each experiment. Generally, the seizure activity was three to five times that of the background activity, and the threshold was set high enough to identify seizure activity only. After the threshold was set for a given experiment, it was not moved. The seizure activity recorded on the field potential traces was directly correlated with behavioral manifestation of the seizures (clonic jerking of the body and forelimbs). When setting the seizure detection threshold, we always verified that the seizure activity identified by the ASD device was directly correlated with this behavioral component of the seizures.

Experimental protocols. The first part of this study was performed to determine whether stimulation of the IO branch of the trigeminal nerve was capable of eliminating PTZ-induced seizure activity in awake rats. To do this, we delivered continuous stimulation to the IO nerve during episodes of PTZ-induced seizure activity via the nerve cuff electrode (Fig. 1 ) for 1 min stimulus-on periods, separated by 1 min stimulus-off periods. This protocol was performed with both unilateral and bilateral stimulation 
of the IO nerve. Stimulus parameters were varied between the stimulus-on periods as described above.

In the second part of this study, we assessed the effectiveness of stimulating the IO nerve only when seizure activity was present by using the ASD. For this protocol, the ASD device was turned on for $1 \mathrm{~min}$ stimulus-on periods, separated by 1 min stimulus-off periods, as in the first protocol, but stimulation was only provided during the stimulus-on periods when seizure activity was detected by the ASD device.

Data analysis. We measured seizure activity in the field potential recordings in three ways: seizure frequency, seizure duration, and integrated seizure activity. These parameters were quantified by the use of a custommade analysis program developed using Matlab. The field potential traces were first bandpass filtered at 5-30 Hz. A sliding window (1 sec window with 0.5 sec overlap) was used to quantify the activity of the absolute values of the field potential traces. Within each window, the amplitude (i.e. voltage) range of the absolute value of the field potential activity in each trace was divided into 10 equal parts, and within each sliding window the number of voltage values falling into each of the 10 divisions of the amplitude range was calculated. Then, a threshold of $50 \%$ of the amplitude range was used to identify seizure activity. If activity within three consecutive windows was above this threshold, the activity was considered to be part of a seizure. From these data, the number of seizures and their durations could be calculated by counting the number of windows during which activity was above the threshold. In addition, a measure we call the "integrated seizure activity" was calculated by summing all of the values for all of the amplitude range intervals for a given on or off period of stimulation. This algorithm was applied in a uniform, blinded manner to all of our data, allowing for objective quantification of the three measures of seizure activity.

Statistical analyses. Using the values for seizure number, seizure duration, and integrated seizure activity, we assessed the efficacy of IO nerve stimulation and ASD by comparing each stimulation-on period with the stimulation-off period directly preceding it. Thus, results are presented as ratios of seizure activity during stimulus-on periods to seizure activity during stimulus-off periods. We used multivariate ANOVAs (MANOVAs) to assess whether there were statistically significant changes in seizure duration, seizure frequency, or integrated seizure activity between periods of no stimulation and periods of stimulation for each stimulus parameter setting. In addition, repeated measure ANOVAs were used when comparing one measure with another (e.g., number of seizures compared with seizure duration). When significant differences were indicated by MANOVA or ANOVA analyses, Tukey's honestly significant difference post hoc tests were used to identify which effects were significant $(p<$ $0.05)$.

\section{RESULTS}

\section{Control experiments}

In control experiments in which PTZ was administered, but no IO nerve stimulation was provided, the average number of seizures per minute was $5.98 \pm 0.45$, and the average seizure duration was $3.94 \pm 0.23 \mathrm{sec}$.

In contrast to studies of VNS in rats (Woodbury and Woodbury, 1990) and dogs (Zabara, 1992), we did not observe any substantial cardiovascular side effects during IO nerve stimulation (Fig. 2). We recorded electrocardiogram (EKG) signals in anesthetized rats while stimulating the IO nerve and did not observe any substantial change in heart rate during stimulation.

\section{Stimulation of the infraorbital nerve reduces seizure activity}

Stimulation of the infraorbital nerve by the use of the periodic stimulation paradigm substantially reduced PTZ-induced seizure activity compared with that of control periods (Figs. 3, 4, 5). This effect was dependent on both the current and the frequency of the stimulation. There were no significant differences between the cortex and thalamus on any of the measures $[\operatorname{Rao} R(3,134)=0.33$; $p>0.8]$.

As expected from previous studies, the seizure reduction effect of IO nerve stimulation was greater with increasing current levels (Fig. $3 b-d$ ). For these experiments, pulse duration and frequency were held constant at $0.5 \mathrm{msec}$ and $333 \mathrm{~Hz}$, respectively, while current was varied between 3 and $11 \mathrm{~mA}$, in $2 \mathrm{~mA}$ increments. At currents of 3 and $5 \mathrm{~mA}$, there were no differences between periods of IO nerve stimulation and periods of no stimulation. However, at 7,9 , and $11 \mathrm{~mA}$, nerve stimulation caused a significant decrease in overall seizure activity (Fig. $3 b, 7 \mathrm{~mA}, 43.2 \pm 7.0 \%$; $9 \mathrm{~mA}, 65.5 \pm$ $4.7 \% ; 11 \mathrm{~mA}, 77.5 \pm 4.3 \% ; p<0.001)$ and in the number of seizures initiated (Fig. $3 c, 7 \mathrm{~mA}, 36.4 \pm 5.8 \%$; $9 \mathrm{~mA}, 50.5 \pm 4.6 \%$;

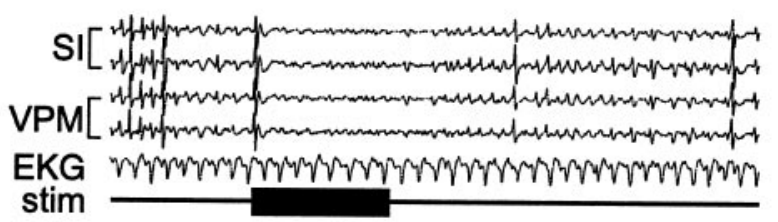

b

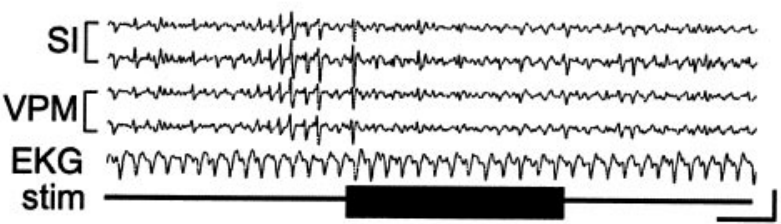

C

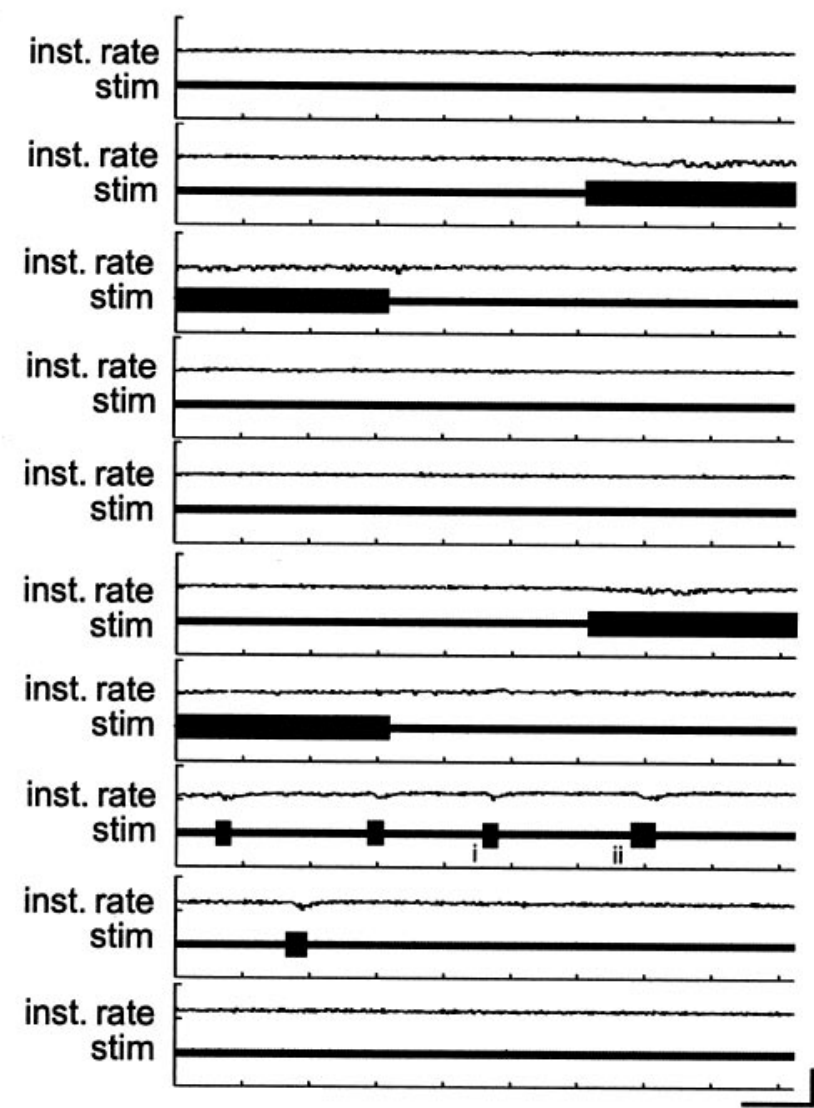

Figure 2. EKG activity is not significantly altered during IO nerve stimulation. $a, b$, Two examples of EKG activity during IO nerve stimulation (stim; horizontal bars) in an anesthetized rat. Calibration: vertical, $100 \mu \mathrm{V}$; horizontal, 1 sec. $c$, The EKG traces and instantaneous heart rate (Inst. rate) over a $15 \mathrm{~min}$ period during which stimulation was twice provided continuously for $1 \mathrm{~min}$, as well as five times for shorter bursts. Small changes in the EKG can be seen when stimulation is provided, but they are minor and rapidly stabilize, even during ongoing stimulation. $i$, $i i$, The traces that are shown at a faster time scale in $a$ and $b$, respectively. Calibration: vertical, $100 \mu \mathrm{V}$ for the EKG traces, 200 beats/min for the instantaneous heart rate; horizontal, $10 \mathrm{sec}$. The stimulus parameters in these traces were $50 \mathrm{~Hz}, 11$ $\mathrm{mA}$, and $0.5 \mathrm{msec}$ pulse duration.

$11 \mathrm{~mA}, 58.7 \pm 6 \% ; p<0.0001)$. There was also a significant decrease in the seizure duration at $9 \mathrm{~mA}$ (Fig. $3 d, 52.5 \pm 3.7 \% ; p<$ $0.0001)$.

Different stimulus frequencies had different effects on the seizure activity (Fig. 4). For these experiments, pulse duration and current were held constant at $0.5 \mathrm{msec}$ and $9 \mathrm{~mA}$, respectively. Stimulation at high frequencies $(100,125,200$, and $333 \mathrm{~Hz})$ caused a significantly smaller number of seizures than did periods of no stimulation (Fig. $4 a ; p<0.05$ ), as described above. Stimulation frequencies of $50 \mathrm{~Hz}$ and lower did not cause any significant changes in the number of seizures initiated (Fig. $4 a ; p=1.0$ ), but seizures did tend 

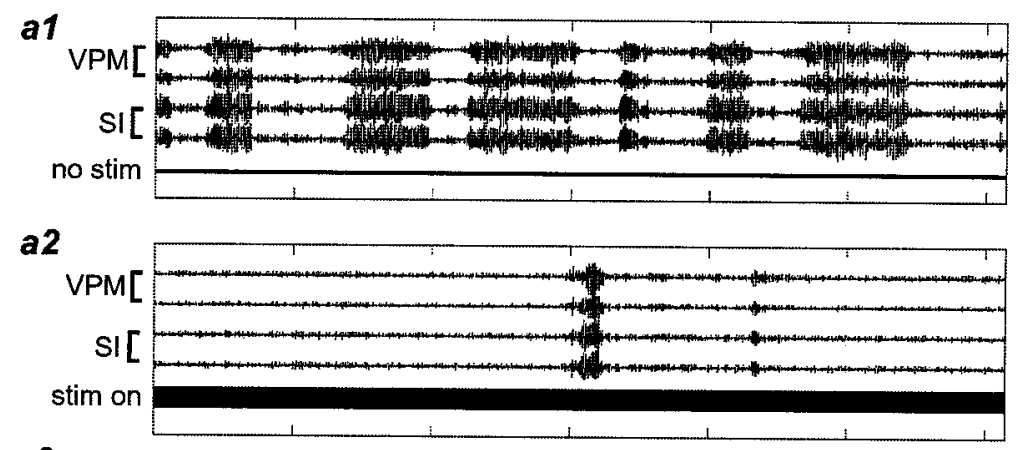

a3
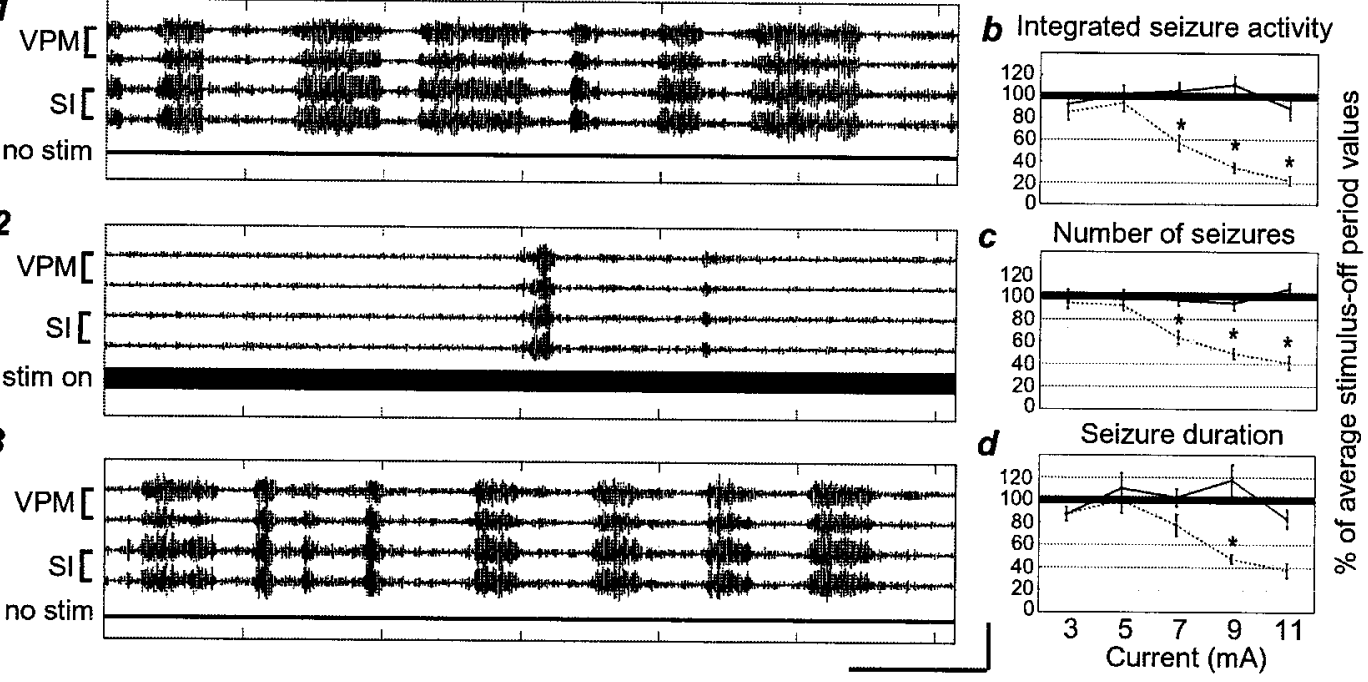

Figure 3. Stimulation of the IO nerve reduces seizure activity in a current-dependent manner. a1-a3, Filtered field potential traces showing seizure activity during three sequential 1 min periods ( $a 1$, no stimulus; $a 2$, stimulus on; $a 3$, no stimulus). The stimulus parameters for this figure were $11 \mathrm{~mA}$, 333 $\mathrm{Hz}$, and 0.5 msec pulse. $b-d$, The amount of seizure activity during 1 min periods of stimulation at different current levels compared with the period of no stimulation directly preceding each stimulus-on period. Values are presented as a percent of the average stimulus-off period measurements. $b$, Integrated seizure activity. $c$, Number of seizures. $d$, Seizure duration. Error bars represent \pm SEM. A solid line connects stimulation-off values; a dashed line connects stimulation-on values. Stimulation-on values significantly different from stimulation-off values are designated by an asterisk. Thick, black horizontal lines at $100 \%$ denote the level of no change in seizure activity. Calibration: vertical, $200 \mu \mathrm{V}$; horizontal, 10 sec.

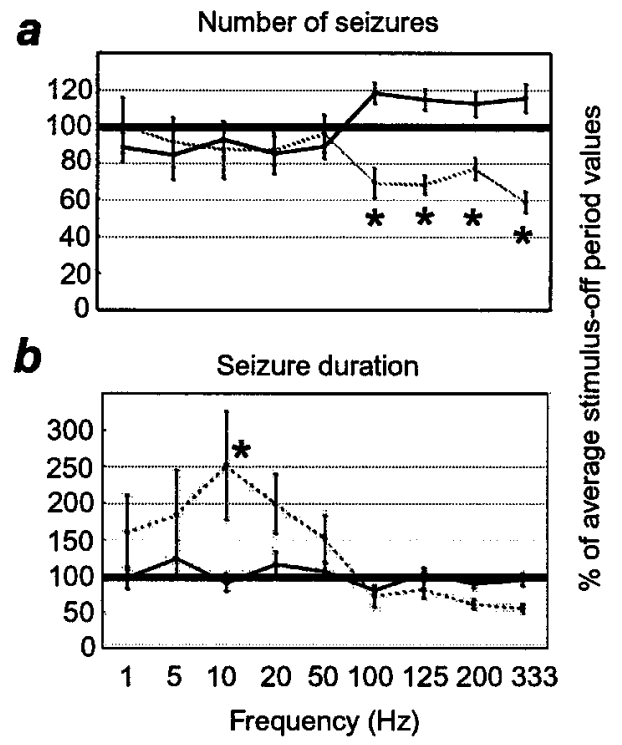

Figure 4. Effect of varying stimulus frequency using the periodic stimulation paradigm. $a$, Number of seizures. $b$, Seizure duration. Labeling conventions are described in Figure 3 (note the change in the scale of the $y$-axis in $b$ ).

to be longer than those during control periods at these frequencies (Fig. $4 b ; 10 \mathrm{~Hz} ; p<0.02$ ).

\section{Bilateral versus unilateral stimulation}

Bilateral stimulation was significantly more effective at reducing seizures than was unilateral stimulation either contralateral or ipsilateral to the recording site (Fig. 5). This effect was significant for the integrated seizure activity measure (Fig. $5 b$ ) at a current level of $7 \mathrm{~mA}(75.7 \pm 5.7 \% ; p<0.002)$, as well as for the number of seizures (Fig. $5 c)$ at 7 and $9 \mathrm{~mA}(7 \mathrm{~mA}, 63.7 \pm 5.3 ; 9 \mathrm{~mA}, 78.1 \pm$ $3.7 \% ; p<0.01)$. It is important to point out that the superior effect of bilateral stimulation was only evident for the middle range of stimulation intensities used in this study. That is, if the current was too low, presumably below the threshold for seizure reduction, there was no advantage in stimulating both nerves, and if the current was high enough, stimulating unilaterally was as effective as stimulating bilaterally. However in the middle range of stimulation intensities, bilateral stimulation allowed us to use less current per nerve while still maintaining a high degree of seizure reduction.

\section{Automatic detection of seizure activity and termination of seizures}

Use of the ASD device to stimulate the IO nerve only when seizure activity was detected successfully reduced the amount of seizure activity relative to control periods. Figure 6 shows that when the seizure detector identified seizure activity in the field potential traces and triggered the stimulator, the seizure stopped. As in the experiments described above, the degree of seizure reduction was dependent on the current level (Fig. 7). For this set of experiments, we held the pulse duration constant at $0.5 \mathrm{msec}$, and the frequency at $333 \mathrm{~Hz}$. Current was varied from 3 to $11 \mathrm{~mA}$ in $2 \mathrm{~mA}$ increments. Figure $7 b$ shows that the integrated seizure activity level was significantly reduced at 9 and $11 \mathrm{~mA}(9 \mathrm{~mA}, 55.2 \pm 7.2 \% ; p<0.03$; $11 \mathrm{~mA}, 56.6 \pm 8.0 \% ; p<0.01)$. The number of seizures was significantly decreased at 7 and $9 \mathrm{~mA}$ (Fig. $7 c, 7 \mathrm{~mA}, 19.3 \pm 5.8 \%$; $p<0.05 ; 9 \mathrm{~mA}, 22.5 \pm 6.1 \% ; p<0.0001)$. In addition, the seizure duration was decreased at 7, 9, and $11 \mathrm{~mA}$ (Fig. $7 d, 7 \mathrm{~mA}, 40.2 \pm$ $3.3 \%$; $9 \mathrm{~mA}, 45.2 \pm 3.6 \%$; $11 \mathrm{~mA}, 49.4 \pm 4.0 \% ; p<0.0001$ for all). To compare the efficacy of the ASD device with that of the periodic stimulation paradigm, we calculated the ratio of the percent of seizure reduction to stimulus-on time (Fig. 8). By comparing these ratios between ASD stimulation and periodic stimulation protocols, we observed that, at least in the acute seizure model (PTZ) used in this study, delivering stimulation only when seizure activity was detected was up to 39.8 times more effective at seizure reduction per second of stimulation than was periodic stimulation not related in any way to seizure activity.

There was an important difference between the nature of the seizure reduction effect using the ASD device and that observed using the periodic stimulation paradigm described above. With the periodic stimulation paradigm, the number of seizures and the seizure durations were reduced by approximately the same amount at each current level (Fig. 3, compare $c, d$ ). However, when the ASD device was used, the seizure durations were reduced significantly more than the number of seizures (Fig. 7, compare $c, d ; p<$ $0.000001)$.

In addition, analysis of the data revealed that in control experi- 


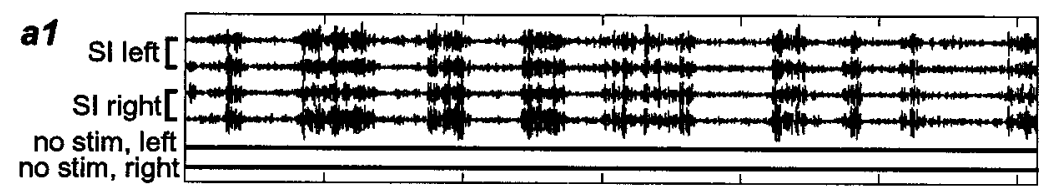

a2

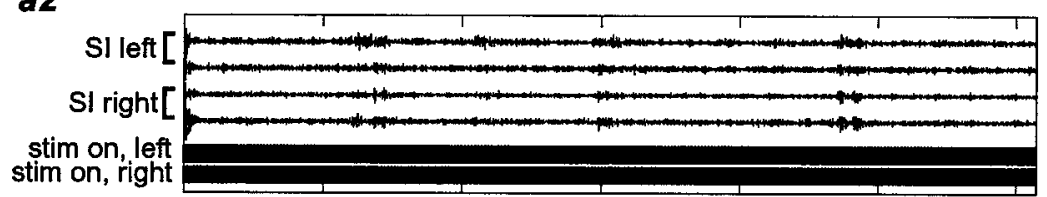

a3

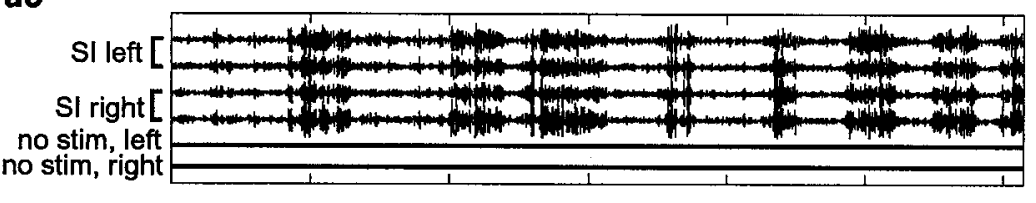

b Integrated seizure activity

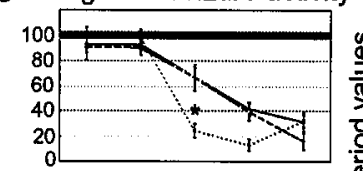

c

Number of seizures

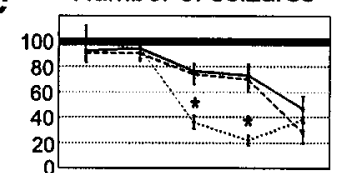

d

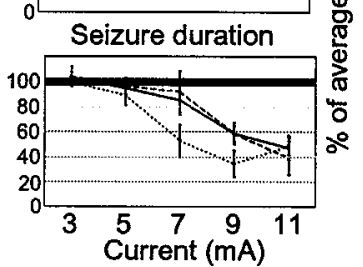

Figure 5. Effects of bilateral stimulation versus unilateral stimulation. a1-a3, Filtered field potential traces showing seizure activity during three sequential 1 min periods ( $a 1$, no stimulus; $a 2$, bilateral stimulation; $a 3$, no stimulus). The stimulus parameters were $9 \mathrm{~mA}, 333 \mathrm{~Hz}$, and $0.5 \mathrm{msec}$ pulse duration. $b-d$, Values presented as ratios of stimulus-on/stimulus-off measurements. $b$, Integrated seizure activity. $c$, Number of seizures. $d$, Seizure duration. A solid line connects responses contralateral to the stimulation site; a line with long dashes connects responses ipsilateral to the stimulation site; a line with short dashes connects responses to bilateral stimulation. Responses to bilateral stimulation that are significantly different from those to ipsilateral and contralateral stimulation are represented by an asterisk. Other labeling conventions are described in Figure 3.

a
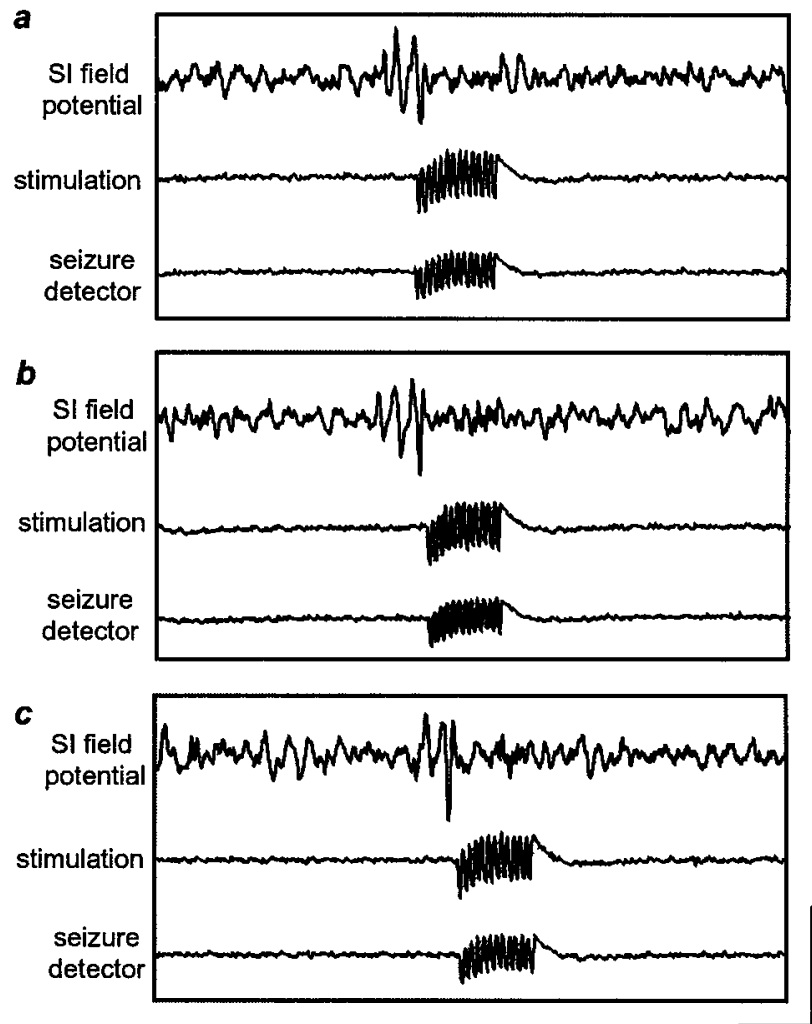

Figure 6. Seizure-specific stimulation stops synchronous activity. When the field potential amplitude reached a threshold value, the seizure detector (ASD device) triggered IO nerve stimulation. $a-c$, Traces correspond to the roman numerals $i$-iii, respectively (see Fig. 7). Note that the stimulation outlasts seizure activity because pulses were provided in $500 \mathrm{msec}$ trains. Also note that the traces indicating seizure detection and stimulation are only indicators and are not indicative of stimulation current or frequency. Calibration: vertical, $200 \mu \mathrm{V}$; horizontal, $500 \mathrm{msec}$.

ments where PTZ was administered but no stimulation was provided, the average time between the end of one spontaneously occurring seizure and the beginning of the next was $6.1 \mathrm{sec}$ (calculated from the average number of seizures and the average seizure duration). We also measured the latency between the end of a stimulus and the next spontaneous seizure (i.e., in the epoch after a stimulus-on period), which was $7.59 \pm 1.29 \mathrm{sec}$. Thus, the average delay between the end of a period of stimulation and the next spontaneously occurring seizure is an average of $24 \%$ longer than the interseizure interval during control experiments where no stimulation was present.

\section{DISCUSSION}

The results of this study demonstrate three substantial advances in the use of cranial nerve stimulation for the treatment of seizures. First, we showed that stimulation of the trigeminal nerve can reduce PTZ-induced seizure activity in rats. This indicates that the seizure reduction effect of cranial nerve stimulation is not limited to stimulation of the vagus nerve but instead may be mediated by a more nonspecific arousal mechanism that can be recruited by stimulation of a number of cranial nerves. Second, we showed that bilateral trigeminal nerve stimulation could have the same seizure reduction effect as unilateral stimulation but required much less current to do so. This finding is therapeutically relevant, because it suggests that multisite stimulation could help maximize the seizure reduction effect of any technique using cranial nerve stimulation, while using the lowest current levels possible. Finally, we showed that in the acute seizure model used in this study (PTZ), automatic, real-time seizure-triggered stimulation reduces seizures more effectively per second of stimulation than does periodic stimulation that is unrelated to seizure onset. This means that the use of a real-time brain-device interface that would automatically detect seizure activity and trigger a nerve stimulator only when such activity was present could provide a high degree of seizure control while potentially reducing the overall amount of stimulation presented to a patient. We propose that these findings may significantly improve the efficacy of cranial nerve stimulation as a therapy for patients with intractable epileptic seizures.

\section{Mechanism of seizure reduction by cranial nerve stimulation}

The mechanism by which cranial nerve stimulation causes desynchronization of thalamic and cortical activity and reduces seizure activity is unknown. However, one theory is that such stimulation activates the midbrain reticular formation and that this activation results in generalized arousal via the reticular-activating system. In support of this view, Gellhorn (1960) showed that stimulation of the midbrain reticular formation suppresses focal strychnine spikes in cats. In addition, several methods of eliminating seizure-related 


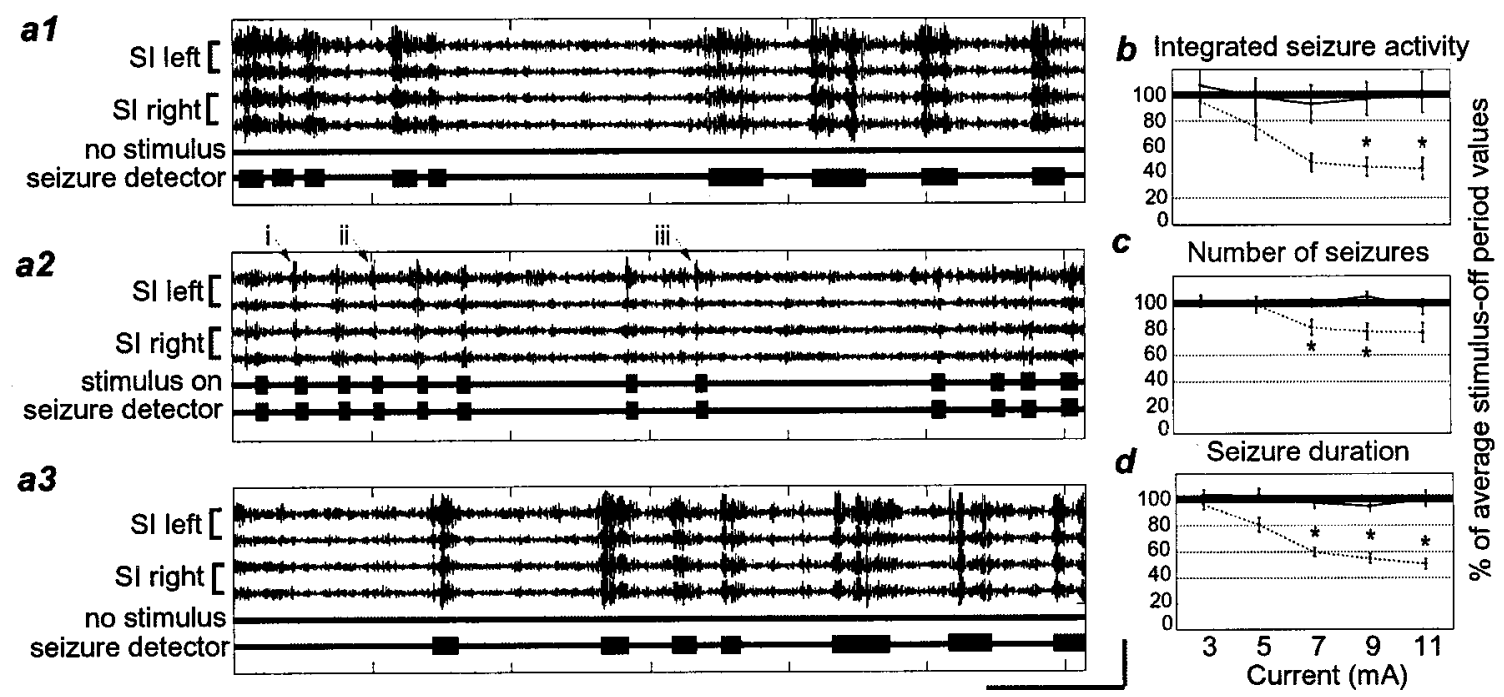

Figure 7. Seizure reduction using the ASD device. a1-a3, Filtered field potential traces showing seizure activity during three sequential 1 min periods (a1, no stimulus; $a 2$, stimulus on; $a 3$, no stimulus). The stimulus parameters were $9 \mathrm{~mA}, 333 \mathrm{~Hz}$, and $0.5 \mathrm{msec}$ pulse duration. Within each segment, the trace labeled seizure detector indicates where the ASD device detected seizure activity; the trace labeled stimulus on indicates where the ASD device sent a TTL pulse to trigger the IO nerve stimulator when it detected such activity. The roman numerals i-iii and arrows indicate parts of the traces that were enlarged to create Figure 6. $b$, Integrated seizure activity. $c$, Number of seizures. $d$, Seizure duration. Labeling conventions are described in Figure 3.

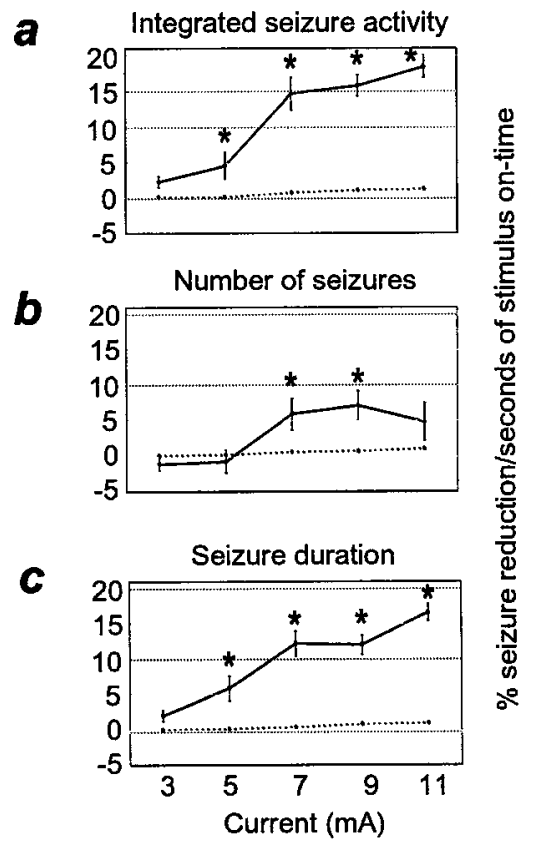

Figure 8. Comparison of the amount of seizure reduction versus the amount of stimulation provided. Stimulation was provided by the use of the periodic stimulation paradigm (dashed line) or the ASD device (solid line). The $y$-axis represents the ratio of seizure activity reduction to seconds of stimulation in a given stimulus-on period. Asterisks designate the ratios of ASD seizure reduction to seconds of stimulation that were significantly higher than those obtained by the use of the periodic stimulation protocol. $a$, Integrated seizure activity. $b$, Number of seizures. $c$, Seizure duration.

activity by activating multiple sensory modalities have been demonstrated. These include the reduction of absence seizures by acoustic stimuli (Rajna and Lona, 1989) and the reduction of interictal focal activity or absence seizures by motor or mental activity (Jung, 1962; Ricci et al., 1972) or by thermal stimulation (McLachlan, 1993). Because such a wide range of manipulations can reduce seizure-related activity, it is reasonable to suggest that seizure reduction in these cases is caused by a generalized effect on arousal mediated by the brainstem reticular formation. This is supported by the classical work of Moruzzi and Magoun (1949) demonstrating that stimulation of the midbrain reticular formation causes EEG desynchronization. This hypothesis is consistent with our finding that seizure reduction effects are not specific to the vagus nerve but can instead be achieved by stimulation of multiple cranial nerves that convey information to the reticular formation.

One important factor to consider with regard to both the mechanism of seizure reduction by trigeminal nerve stimulation and its applicability to long-term use in humans is the nature of the fiber types that must be activated to cause the seizure reduction effect. Multiple studies of the VNS technique have shown that the level of stimulation, in terms of stimulus frequency and intensity, must be high enough to activate slowly conducting c-fibers (Chase et al., 1967; Woodbury and Woodbury, 1990). The frequency range we found to be therapeutic in the present study was somewhat different from that typically used in animal and human VNS studies. In animal studies the usual therapeutic range was generally $10-30 \mathrm{~Hz}$ (Woodbury and Woodbury, 1990; Zabara, 1992; Takaya et al., 1996), although Lockard et al. (1990) used higher stimulation frequencies $(50-250 \mathrm{~Hz})$ in monkeys. In human studies the range used for stimulation was typically 20-30 Hz (McLachlan, 1997). This difference between VNS studies and ours may be caused by the difference in the relative numbers of fiber types between the vagus nerve and the infraorbital nerve. In cat, the vagus nerve is composed of 65-90\% unmyelinated fibers (Foley and DuBois, 1937; Agostoni et al., 1957), whereas the rat IO nerve contains $\sim 33 \%$ slowly conducting, unmyelinated fibers (Klein et al., 1988). However, it is not clear what the relationship is between fiber composition and the stimulus frequency/intensity required for seizure reduction, so interpreting these differences is difficult. A complicating factor is that although it has been shown that for seizure reduction the level of stimulation must be sufficient to activate c-fibers, it has not been demonstrated that these fibers are necessary for the seizure reduction effect. Finally, it is important to note that according to studies by Torebjork and colleagues (Torebjork, 1974; Torebjork and Hallin, 1974), c-fibers do not conduct if electrical stimuli are presented at frequencies above $\sim 10 \mathrm{~Hz}$. This means that although high stimulation frequencies were required for the seizure reduction effect observed here, it is likely that at such frequencies the c-fibers were not activated or were activated to a lesser degree than other fibers in the nerve.

Furthermore, it is possible that cells in the trigeminal nucleus were not able to follow with sustained responses at the high rates of stimulation we provided. For example, Andresen and Yang (1995) 
demonstrated using a slice preparation of the rat medulla that neurons in the nucleus of the solitary tract (NTS) responded with lower EPSP amplitudes as the frequency of solitary tract stimulation was increased. These results may also be relevant to trigeminal nerve stimulation. If this is the case, it is unclear why our results show that higher frequency stimulation is more effective for seizure elimination than are lower stimulation rates. However, the study by Andresen and Yang (1995) also demonstrated that bursts of highfrequency stimulation resulted in less EPSP attenuation than did continuous high-frequency stimulation, suggesting that an optimal stimulation protocol could involve short bursts of high-frequency stimulation rather than continuous trains.

The delay between the onset of seizure-triggered stimulation and the end of the seizure activity might shed some light on the mechanism by which trigeminal stimulation reduces seizure activity. The average time between the onset of the seizure-triggered stimulus and the end of the seizure was $529.9 \pm 40.3$ msec (note that Fig. 6 demonstrates some of the shortest delays). It is interesting that this value is similar to the minimum effective stimulus train duration $(500 \mathrm{msec})$ that we determined empirically. However, it should be noted that there was a wide range of delays, and this may be caused by at least two factors. First, the phase of the synchronous oscillations during which the stimuli occur may have a profound impact on the efficacy of the stimulation. Second, it is possible that the ability to abort a seizure varies depending on the amount of time the seizure has been ongoing before a sufficient stimulus arrives. Thus, differences in the phase of the oscillatory seizure activity at which the stimuli occur or the threshold used for seizure detection may affect the efficacy of the stimulation. These mechanisms could explain the variation in the amount of time required to abort a seizure.

Another important mechanism-related issue is whether the trigeminal stimulation was merely able to stop seizure activity during the stimulation itself or whether it also had an effect on the number of seizures initiated. In control files where PTZ was administered but no stimulation was provided, the average time between the end of one spontaneously occurring seizure and the beginning of the next was $6.1 \mathrm{sec}$ (calculated from the average number of seizures and the average seizure duration, as reported in Results). We also measured the latency between the end of a period of stimulation and the next spontaneous seizure (i.e., in the epoch after a stimulus-on period), which was $7.59 \pm 1.29 \mathrm{sec}$. Thus, the average delay between the end of a period of stimulation and the first spontaneous seizure after the stimulus ends is actually, on average, $24 \%$ longer than the interseizure interval during control files with no stimulation present. These results are supported by results from other laboratories (Zabara, 1992; Takaya et al., 1996) showing that the seizure reduction effect of vagus nerve stimulation can outlast the stimulus duration.

\section{Bilateral versus unilateral IO nerve stimulation}

The fact that bilateral stimulation can be more effective than unilateral stimulation in the middle of the therapeutic-current range has implications for how such stimulation could be used to most effectively reduce seizure activity. Specifically, because bilateral stimulation at $7 \mathrm{~mA}$ was just as effective as unilateral stimulation at $11 \mathrm{~mA}$ (Fig. 5), the use of bilateral nerve cuff electrodes would reduce the amount of current delivered to each nerve, while still maintaining the same seizure reduction effect as higher stimulation current at a single site. This would be beneficial because it would reduce the potential for damage to nerve fibers at the stimulation site (Agnew et al., 1989; Agnew and McCreery, 1990), and it would reduce the intensity of any possible side effects associated with the stimulation. Bilateral stimulation is a further improvement over VNS, because the vagus nerve cannot be safely stimulated bilaterally without substantial risk of cardiovascular side effects (Schachter and Saper, 1998).

It is important to point out that our finding that bilateral stimulation of the IO nerve was more effective than unilateral stimulation is in contrast to two previous studies reporting that bilateral stimulation of the vagus nerve was no more effective than unilateral stimulation (Chase et al., 1966; Zabara, 1992). This discrepancy is likely either caused by differences in fiber composition between the vagus nerve and the IO nerve or caused by the fact that the stimulus parameters used in those studies were beyond those for which bilateral stimulation is superior to unilateral stimulation. Details about the stimulus parameters used for assessing the efficacy of bilateral stimulation in those two studies were not provided.

Another important point to consider is that we have tested the effect of bilateral stimulation with the PTZ seizure model, which involves generalized, tonic-clonic seizures (Fisher, 1989). Further testing with focal seizure models such as localized application of alumina gel (Lockard et al., 1990) or penicillin (McLachlan, 1993) to the cortex will be necessary to determine whether there is an advantage to bilateral stimulation in eliminating these types of seizures as well. Evidence to support an advantage in using bilateral stimulation to treat focal seizures is that, in our study, unilateral stimulation eliminated seizure activity in both hemispheres at the same time, suggesting that the effect of the stimulation is not restricted to one hemisphere. Such results have also been found for VNS in cats (Chase et al., 1966), dogs (Zabara, 1992), and humans (Henry et al., 1998, 1999). These results suggest that because each nerve being stimulated can reduce seizures bilaterally, the effect of stimulating both nerves could be additive within a given hemisphere.

\section{A brain-device interface for automatic, real-time detection and reduction of seizure activity}

This study showed that triggering trigeminal nerve stimulation only when a seizure began is a much more effective method for reducing seizure activity than is providing stimulation on a fixed duty cycle, as has been used in past studies. This finding is an important advancement in the use of cranial nerve stimulation therapies in epilepsy for several reasons.

First, stimulating only when seizure activity occurs would, for many patients, reduce the overall amount of stimulation required for maintaining seizure control. Thus, the amount of potentially unnecessary stimulation usually occurring between seizure periods would be reduced, decreasing the possibility of damage to the nerve (Agnew et al., 1989; Agnew and McCreery, 1990; Ramsay et al., 1994). It is important to note, however, that several researchers have demonstrated a prophylactic effect of vagus nerve stimulation such that after stimulation, seizures are less likely for a period of time related to the duration of the preceding stimulation (Zabara, 1992; Takaya et al., 1996). This implies that perhaps the best overall treatment stimulation protocol might involve the use of seizuretriggered stimulation combined with intermittent prophylactic nonseizure-triggered stimulation.

The second advantage of this technique is that it would reduce the side effects experienced by patients when the stimulus is on. For example, patients undergoing VNS treatment report hoarseness, coughing, and throat pain as the most common side effects of the stimulation (Ramsay et al., 1994; McLachlan, 1997; Schachter and Saper, 1998). These side effects are generally only experienced when the stimulation is on. However, if stimulation were only presented in response to the detection of seizure activity (or occasionally prophylactically, as described above), these side effects would be experienced as infrequently as possible.

In the future, the type of real-time, automatic seizure detector described here could be implemented in humans by building on and combining a number of existing technologies. First, seizure detection could be performed by a computer microchip programmed with a seizure detection algorithm and carried by the patient, similar to the Holter monitors used for continuous EKG monitoring. Input would be delivered to this microchip from multiple scalp EEG electrodes that would be able to pick up and amplify extracranial EEG signals. Finally, when the microchip detected seizure activity in the EEG signals, it would trigger an implanted stimulator similar to those used in the VNS technique (Terry et al., 1990), which would stimulate one or more trigeminal nerve cuff elec- 
trodes. This device would function in a manner analogous to cardiac pacemakers, commonly used to treat heart arrhythmia, and would require a minimum of invasive procedures. In essence, this device would constitute a "brain pacemaker" for seizure monitoring and control.

The application of nonlinear computational methods for detection of seizure activity (Gabor et al., 1996; Webber et al., 1996) could be extremely beneficial if incorporated into the seizure detector described here. Such seizure detection algorithms would allow for more accurate identification of seizure activity than the rather simple amplitude-based algorithm we used in this study.

Another substantial advance could be in the implementation of seizure prediction algorithms that can identify seizures seconds or minutes before the behavioral onset (Martinerie et al., 1998; Le Van Quyen et al., 1999). There is evidence that the sooner stimulation is provided after a seizure begins, the more effectively the seizure can be stopped (Uthman et al., 1993); also stimulation is more likely to prevent seizure activity if it is presented before rather than after a seizure has begun (Woodbury and Woodbury, 1990). Therefore, it is possible that providing stimulation before the clinically defined onset of a seizure may prevent seizures before they begin or become behaviorally relevant to the patient. Such a technique could dramatically improve the efficacy of the cranial nerve stimulation therapy.

\section{Application to human patients}

Further studies in other animals and with other seizure models will be needed to determine whether it is appropriate to apply the techniques described here to human patients and what the best methods for doing so would be.

The results presented here apply to seizures caused by systemic administration of PTZ, which is a model of acute, generalized, tonic-clonic seizures. It will be important to determine whether the results also apply to chronic seizure models, as well as to other seizure types, such as focal seizures (e.g., temporal lobe seizures) and absence seizures, if the described techniques are to be applicable to humans.

Because the cellular mechanisms involved in different seizure types may not necessarily be similar, it is vital to ask whether the seizure reduction effects from trigeminal nerve stimulation would apply to different seizure types. If the effects of trigeminal nerve stimulation are spatially restricted or only affect certain types of cellular excitation or inhibition, then this technique may be of limited use in treating multiple seizure types. If, however, as proposed in the current paper, the mechanism by which trigeminal nerve stimulation reduces seizure activity is, indeed, a generalized, widespread effect on cortical arousal level, perhaps mediated by the brainstem reticular-activating system, it is possible that this technique would be useful in treating a wide range of seizures. In support of this view, the VNS technique has proven to be effective in multiple seizure models including intraperitoneal injection of PTZ (Zabara, 1985, 1992; Woodbury and Woodbury, 1990), intraperitoneal injection of 3-mercaptopropionate (Woodbury and Woodbury, 1990), maximal electroshock (Woodbury and Woodbury, 1990), topical application of penicillin to the cortex (McLachlan, 1993), and chronic local application of alumina gel (Lockard et al., 1990).

Another issue that will need to be addressed before this technique is applied to humans is that because the trigeminal nerve is involved in transmitting both somatosensory and pain information from the head, it is vital that the level of stimulation be below that that might cause discomfort such as facial pain or headaches. It is not known what stimulus parameters would be required to achieve seizure reduction without resulting in painful sensations such as these. However, such side effects could be substantially reduced by using the lowest effective stimulus parameters, which would be aided by the use of bilateral stimulation.

Finally, it would also be possible to develop an effective therapy by combining the VNS technique, which is currently in use in human patients, with the automatic seizure detection technique described in this paper.

\section{Conclusions}

The results described in this study could serve to substantially increase the efficacy of cranial nerve stimulation as a technique for reducing or eliminating seizures in patients who suffer from intractable epilepsy. Further development and testing of trigeminal nerve stimulation for patients with epilepsy is justified on the basis of the results presented here. In addition, our findings suggest that in the future, it will be feasible to develop a completely implantable and relatively noninvasive brain-device interface capable of automatically detecting seizure activity and triggering stimulation of cranial nerves to safely and efficiently reduce seizure activity.

\section{REFERENCES}

Agnew WF, McCreery DB (1990) Considerations for safety with chronically implanted nerve electrodes. Epilepsia 31[Suppl 2]:S27-S32.

Agnew WF, McCreery DB, Yuen TG, Bullara LA (1989) Histologic and physiologic evaluation of electrically stimulated peripheral nerve: considerations for the selection of parameters. Ann Biomed Eng 17:39-60.

Agostoni E, Chinnock JE, De Burgh Daly M, Murray JG (1957) Functional and histological studies of the vagus nerve and its branches to the heart, lungs and abdominal viscera in the cat. J Physiol (Lond) 135:182-205.

Andresen MC, Yang M (1995) Dynamics of sensory afferent synaptic transmission in aortic baroreceptor regions of nucleus tractus solitarius. J Neurophysiol 74:1518-1528.

Ben-Menachem E, Manon-Espaillat R, Ristanovic R, Wilder BJ, Stefan H, Mirza W, Tarver WB, Wernicke JF (1994) Vagus nerve stimulation for treatment of partial seizures: 1 . A controlled study of effect on seizures. First International Vagus Nerve Stimulation Study Group. Epilepsia 35:616-626.

Chase MH, Nakamura Y (1968) EEG response to afferent abdominal vagal stimulation. Electroencephalogr Clin Neurophysiol 24:396.

Chase MH, Sterman MB, Clemente CD (1966) Cortical and subcortical patterns of response to afferent vagal stimulation. Exp Neurol 16:36-49.

Chase MH, Nakamura Y, Clemente CD, Sterman MB (1967) Afferent vagal stimulation: neurographic correlates of induced EEG synchronization and desynchronization. Brain Res 5:236-249.

Fanselow EE, Nicolelis MA (1999) Behavioral modulation of tactile responses in the rat somatosensory system. J Neurosci 19:7603-7616.

Fisher RS (1989) Animal models of the epilepsies. Brain Res Rev 14:245-278.

Foley JO, DuBois F (1937) Quantitative studies of the vagus nerve in the cat. J Comp Neurol 67:49-67.

Gabor AJ, Leach RR, Dowla FU (1996) Automated seizure detection using a self-organizing neural network. Electroencephalogr Clin Neurophysiol 99:257-266.

Gellhorn E (1960) Further experiments on the influence of afferent stimulation on cortical strychnine discharges. Electroencephalogr Clin Neurophysiol 12:613-619.

Henry TR, Bakay RA, Votaw JR, Pennel PB, Epstein CM, Faber TL, Grafton ST, Hoffman JM (1998) Brain blood flow alterations induced by therapeutic vagus nerve stimulation in partial epilepsy: I. Acute effects at high and low levels of stimulation. Epilepsia 39:983-990.

Henry TR, Votaw JR, Pennel PB, Epstein CM, Bakay RA, Faber TL, Grafton ST, Hoffman JM (1999) Acute blood flow changes and efficacy of vagus nerve stimulation in partial epilepsy. Neurology 52:1166-1173.

Jung R (1962) Blocking of petit-mal attacks by sensory arousal and inhibition of attacks by an active change in attention during the epileptic aura. Epilepsia 3:435-437.

Klein BG, Renehan WE, Jacquin MF, Rhoades RW (1988) Anatomical consequences of neonatal infraorbital nerve transection upon the trigeminal ganglion and vibrissa follicle nerves in the adult rat. J Comp Neurol 268:469-488.

Le Van Quyen M, Martinerie J, Baulac M, Varela F (1999) Anticipating epileptic seizures in real time by a non-linear analysis of similarity between EEG recordings. NeuroReport 10:2149-2155.

Lockard JS, Congdon WC, DuCharme LL (1990) Feasibility and safety of vagal stimulation in monkey model. Epilepsia 31[Suppl 2]:S20-S26.

Magnes J, Moruzzi G, Pompeiano O (1961) Synchronization of the EEG produced by low-frequency electrical stimulation of the region of the solitary tract. Arch Ital Biol 99:33-67.

Martinerie J, Adam C, Le Van Quyen M, Baulac M, Clemenceau S, Renault B, Varela FJ (1998) Epileptic seizures can be anticipated by non-linear analysis [see comments]. Nat Med 4:1173-1176.

McLachlan RS (1993) Suppression of interictal spikes and seizures by stimulation of the vagus nerve. Epilepsia 34:918-923.

McLachlan RS (1997) Vagus nerve stimulation for intractable epilepsy: a review. J Clin Neurophysiol 14:358-368.

McNamara JO (1999) Emerging insights into the genesis of epilepsy. Nature 399[Suppl 6738]:A15-A22. 
Moruzzi G, Magoun HW (1949) Brainstem reticular formation and activation of the EEG. Electroencephalogr Clin Neurophysiol 1:455-473.

Nicolelis MA, Ghazanfar AA, Faggin BM, Votaw S, Oliveira LM (1997) Reconstructing the engram: simultaneous, multisite, many single neuron recordings. Neuron 18:529-537.

Paxinos G, Watson C (1986) The rat brain, Ed 2. New York: Academic, Harcourt, Brace, and Jovanovich.

Penry JK, Dean JC (1990) Prevention of intractable partial seizures by intermittent vagal stimulation in humans: preliminary results. Epilepsia 31[Suppl 2]:S40-S43.

Rajna P, Lona C (1989) Sensory stimulation for inhibition of epileptic seizures. Epilepsia 30:168-174.

Ramsay RE, Uthman BM, Augustinsson LE, Upton AR, Naritoku D, Willis J, Treig T, Barolat G, Wernicke JF (1994) Vagus nerve stimulation for treatment of partial seizures: 2. Safety, side effects, and tolerability. First International Vagus Nerve Stimulation Study Group. Epilepsia 35:627-636.

Ricci G, Berti G, Cherubini E (1972) Changes in interictal focal activity and spike-wave paroxysms during motor and mental activity. Epilepsia 13:785-794.

Schachter SC, Saper CB (1998) Vagus nerve stimulation. Epilepsia 39: 677-686.

Takaya M, Terry WJ, Naritoku DK (1996) Vagus nerve stimulation induces a sustained anticonvulsant effect. Epilepsia 37:1111-1116.

Terry R, Tarver WB, Zabara J (1990) An implantable neurocybernetic prosthesis system. Epilepsia 31[Suppl 2]:S33-S37.

Torebjork HE (1974) Afferent C units responding to mechanical, thermal and chemical stimuli in human non-glabrous skin. Acta Physiol Scand 92:374-390.

Torebjork HE, Hallin RG (1974) Responses in human A and C fibres to repeated electrical intradermal stimulation. J Neurol Neurosurg Psychiatry 37:653-664.

Uthman BM, Wilder BJ, Hammond EJ, Reid SA (1990) Efficacy and safety of vagus nerve stimulation in patients with complex partial seizures. Epilepsia 31[Suppl 2]:S44-S50.

Uthman BM, Wilder BJ, Penry JK, Dean C, Ramsay RE, Reid SA, Hammond EJ, Tarver WB, Wernicke JF (1993) Treatment of epilepsy by stimulation of the vagus nerve. Neurology 43:1338-1345.

Vagus Nerve Stimulation Study Group (1995) A randomized controlled trial of chronic vagus nerve stimulation for treatment of medically intractable seizures. Neurology 45:224-230.

Webber WR, Lesser RP, Richardson RT, Wilson K (1996) An approach to seizure detection using an artificial neural network (ANN). Electroencephalogr Clin Neurophysiol 98:250-272.

Woodbury DM, Woodbury JW (1990) Effects of vagal stimulation on experimentally induced seizures in rats. Epilepsia 31[Suppl 2]:S7-S19.

Zabara J (1985) Time course of seizure control to brief, repetitive stimuli. Epilepsia 26:518.

Zabara J (1992) Inhibition of experimental seizures in canines by repetitive vagal stimulation. Epilepsia 33:1005-1012.

Zanchetti A, Wang SC, Moruzzi G (1952) The effect of vagal afferent stimulation on the EEG pattern of the cat. Electroencephalogr Clin Neurophysiol 4:357-361. 